\title{
Quantifying, predicting, and exploiting uncertainties in marine environments
}

\author{
Michel Rixen • Pierre Lermusiaux • John Osler
}

Received: 13 January 2012 / Accepted: 23 January 2012 /Published online: 15 February 2012

(C) The Author(s) 2012. This article is published with open access at Springerlink.com

\section{Overview}

Following the scientific, technical, and field trial initiatives ongoing since the Maritime Rapid Environmental Assessment (MREA) conferences in 2003, 2004, and 2007, the MREA10 conference provided a timely opportunity to review the progress on various aspects of MREA, with a particular emphasis on marine environmental uncertainty management. A key objective of the conference was to review the present state of the art in quantifying, predicting, and exploiting marine environmental uncertainties. The integration of emerging environmental monitoring and modeling techniques into data assimilation streams and their subsequent exploitation at an operational level involves a complex chain of nonlinear uncertainty transfers, including human factors. Accordingly, the themes for the MREA10 conference were selected to develop a better understanding of uncertainty, from its inception in the properties being measured and instrumentation employed to its eventual impact in the applications that rely upon environmental information.

\section{Responsible Editor: Jörg-Olaf Wolff}

\section{Rixen $(\bowtie)$}

World Meteorological Organization,

Geneva, Switzerland

e-mail: mrixen@wmo.int

P. Lermusiaux

Massachusetts Institute of Technology,

Room 5-207B, 77 Massachusetts Avenue,

Cambridge, MA 02139-4307, USA

e-mail: pierrel@mit.edu

URL: http://web.mit.edu/pierrel/www/

\section{J. Osler}

NATO Undersea Research Center (NURC),

Viale San Bartolomeo, 400,

19126 La Spezia, Italy

e-mail: osler@nurc.nato.int
Contributions from the scientific community were encouraged on all aspects of environmental uncertainties: their quantification, prediction, understanding, and exploitation. Contributions from operational communities, the consumers of environmental information who have to cope with uncertainty, were also encouraged. All temporal and spatial scales were relevant: tactical, operational, and strategic, including uncertainty studies for topics with long-term implications. Manuscripts reporting new technical and theoretical developments in MREA, but acknowledging effects of uncertainties to be accounted for in future research, were also included.

The response was excellent with 87 oral presentations (11 of which were invited keynote speakers) and 24 poster presentations during the conference. A subset of these presentations was submitted to this topical issue, and 22 manuscripts were published by Ocean Dynamics. The following section includes an overview of the conference themes and summary of the published manuscripts.

\section{Conference themes and findings}

2.1 Quantify: review and quantify contributions to marine environmental uncertainty

This theme considered sensor and platform related uncertainties from: satellite remote sensing, coastal and marine radars, autonomous vehicles (gliders, AUVs), representation errors, and calibration/validation.

Pleskachevsky et al. (2011) present a new approach for bathymetry estimation from combined optical and synthetic aperture radar data covering two different depth domains. The overlapping range from 20 up to $10 \mathrm{~m}$ provides some perspectives on fusing the results from the two approaches. Underwater topography is derived using the shallow water 
dispersion relationship. Results are found to depend on the image acquisition quality and sea state.

Bell and Osler (2011) investigate the potential of marine radar mounted on moving vessels in mapping large areas of shallow or coastal seas prior to the surveying vessel actually having to travel into potentially uncharted or dangerous shallow water areas. Results of bathymetry analyses of two datasets are presented while the vessel was traveling at speeds of up to 14 knots. The bathymetry derived from the radar data compare favorably with independent surveys and with the on-board echo sounder.

Holman et al. (2011) investigate methods and issues for estimating nearshore bathymetry based on wave celerity measurements obtained using time series imagery from small unmanned airborne systems. Although these time series are usually short and have gaps, it has been found that records as short as $50 \mathrm{~s}$ or less can yield good bathymetry results.

Shen et al. (2012) present two new methods to invert wind speed from HF radar first-order backscatter signal. The relationship between wind speed and wave generation at the Bragg frequency matching that of the radar is explored using artificial neural network analysis to derive the interdependence of the relevant parameters. The results show that the methods require minimum radar-frequencydependent wind speeds and are limited by wave energy saturation at the Bragg wave frequency.

Wadhams (2012) discusses the use of autonomous underwater vehicles (AUVs) to map the variability of under-ice topography. The images acquired from both large and small vehicles were found to be complementary and have shown that although first-year ridges have the familiar shape of a triangular prism made of small ice blocks, multi-year ridges are found to be broken up by lead formation into a chain of individual large ice blocks rather than a coherent linear feature.

Brizzolara et al. (2011) present an innovative platform conceived and designed to extend the operational capabilities of current unmanned surface vehicles in terms of platform stability in waves and of powering requirement at a relatively high speed. The choice of a Small Waterplane Area Twin Hull form has been motivated by the inherent seakeeping qualities, combined with a nonconventional low resistance underwater hull shape, whose geometric parameters have been optimized with a state-of-the-art computational fluid dynamics flow solver and a differential evolution global minimization algorithm.

Chiggiato et al. (2012) report on Maritime Rapid Environmental Assessment results from the Turkish Straits System experiments coordinated by the NATO Undersea Research Centre in September 2008 and February 2009. The authors focus on data-driven numerical experiments using the Regional Ocean Modeling System for modeling the observed variability of the Marmara Sea upper layer circulation at mesoscale resolution over the entire basin. They confirm a westward meandering current associated with a recurrent anticyclone. They also study the roles of varied dynamical factors on the mesocale features, emphasizing the effects of wind stress curl and strait flows.

2.2 Predict: investigate ways to robustly model and predict uncertainties

This theme considered modeling and data assimilation uncertainties, including: deterministic and non-deterministic approaches, model error covariances, ensemble and multimodel forecasting, and coupled modeling.

Holt et al. (2011) describe the coupled ocean-atmosphere mesoscale ensemble prediction system developed by the Naval Research Laboratory and its application to coupled ensemble simulations for two tropical cyclones. The system can account for all major sources of uncertainties. Its components include the Coupled Ocean/Atmosphere Mesoscale Prediction System, the Navy Coastal Ocean Model for atmosphere and ocean forecasting, the NRL Atmospheric Variational Data Assimilation System, and the Navy Coupled Ocean Data Assimilation for atmosphere and ocean data assimilation. The ensembles are generated using separate applications of the Ensemble Transform technique in both the atmosphere and the ocean. Results for Hurricanes Hanna and Ike show that the coupled ensemble can provide useful uncertainty information about the storm track and intensity.

Mourre et al. (2011) report on the performance of the 3-D super-ensemble technique to forecast ocean temperature and associated uncertainty in the Ligurian Sea. The temperature uncertainty estimate is obtained using the product of the a posteriori model weight error covariances and an operator containing model forecast values. The 3DSE is found to underestimate uncertainty during the forecast period; thus, a time-evolving "calibration" factor is introduced to account for the influence of ocean dynamics and inaccuracies in the a priori weight error correlations.

Bertotti and Cavaleri (2011) have explored the predictability of storms in a small enclosed basin. Comparisons with surface winds and wave heights for some major events have demonstrated robust predictability $(10 \%$ relative error) up to day 4 , and growing errors (up to $30 \%$ ) at day 6.

Breivik et al. (2011) investigate an original approach of backtracking drifting objects to determine their origin. Instead of running a trajectory model in reverse which is inherently limited by nonlinearities, the original (forward) trajectory model is kept unaltered, while an iterative seeding and selection process allows the retention of only those particles that end up within a certain time-space radius of 
the observation. The backtracking method is tested against the drift trajectory of a life raft and is shown to predict closely the initial release position of the raft and its subsequent trajectory.

Vousdoukas et al. (2012) perform a coastal vulnerability assessment based on video wave run-up observations at a meso-tidal, steep-sloped beach. The time-stacked images were processed in an open-access, freely available Graphical User Interface software, developed to extract and process time series of the cross-shore position of the swash extrema and develop empirical formulas. The equation was applied to predict overwash for four extreme storm scenarios. The prediction capacity improved when the shore normal wind speed component and/or the tidal elevation were included in the parameterizations.

Marmain et al. (2011) discuss two methods to extract the ocean surface circulation based on a single HF/VHF radar station, assuming a nondivergent horizontal surface current. Results based on simulated and real data sets demonstrated the successful detection of a vortex in the Gulf of Lions but suggest that the underlying working hypothesis is by far the most limiting factor.

\subsection{Understand: understand process level and transfers of uncertainties}

This theme considered uncertainty processes and transfers, including: parameterization, air-sea interaction, turbulence, case studies, transfer of uncertainties, irreducible uncertainties, and "uncertainties of uncertainties."

Callies et al. (2011) consider marine data from the Station Helgoland Roads in the southeastern North Sea (German Bight) and study how simulations of transport and water mass exchanges for the region depend on the choice of a specific hydrodynamic model. The authors complete a large set of Lagrangian simulations within the period February 2002-October 2004, using four different models of diverse complexity. They find systematic differences between drift simulations, but could not assign these differences to specific attributes such as spatial resolution or baroclinic terms. The authors nonetheless recommend multi-model simulations for uncertainty assessments and long-term predictions.

Blain et al. (2011) study the predictive capabilities and uncertainties of a limited-domain coastal ocean forecasting system at predicting near-coastal currents at the mouth of Chesapeake Bay during a Navy exercise. The forecasting system consists of an unstructured grid nearshore ocean model, a meteorological model, a regional ocean model, and a global tidal database. Two-day forecasts are compared to ocean data for a 10-day period from 04-14 June 2010 . The authors show that the spatial resolution of the regional model does not significantly affect the skill. They indicate that the dominant M2 tide is well represented but that diurnal tides, especially $\mathrm{K} 1$, are not. The authors suggest that sufficient resolution of the wind forcing, especially in areas close to the shoreline, is critical for accurate nearshore forecasts.

Pecknold and Osler (2011) present an approach to quantify and manage environmental uncertainty using sensitivity metrics and Monte Carlo simulations of acoustic propagation with multiple realizations of the marine environment. This approach can be simplified by using a linearized twopoint sensitivity measure based on the statistics of the environmental parameters used by acoustic propagation models. Forecasts and in situ data are used with historical geoacoustic databases and geo-acoustic parameters collected using Rapid Environmental Assessment techniques to perform acoustic transmission loss predictions, which are then compared to measured transmission loss. They discuss the progression of uncertainties in various marine environments and the consequences on acoustic propagation.

Hjelmervik et al. (2011) report on a technique to identify acoustically "stable" geographical regions by applying empirical orthogonal functions to oceanographic sound speed profiles and grouping algorithms. The stability is first evaluated in an oceanographic context using an empirical orthogonal functions (EOF) cost function to capture oceanographic variations. Then, because there is no linear relationship between sonar conditions and these oceanographic variations, they introduce an acoustic cost function for specific sonar systems and geometries that is evaluated as a function of the EOF cost function. The resulting maps of nowcast and forecast acoustic stability are an MREA product in and of themselves, or may be used to indicate regions in which additional sampling would be beneficial to reduce uncertainty.

Cococcioni et al. (2012) discuss oil spill detection from remotely sensed optical images and the automatic classification of regions of interest (ROIs) in two classes, namely oil spills or look-alikes. A set of features are extracted from each ROI, allowing the oil spill detection problem to be formulated as a two-class classification task using a supervised learning strategy. This is based on an ensemble of incremental and decremental cost-oriented support vector machines, aggregated with the receiving operating characteristic (ROC) convex hull method in the ROC space.

\subsection{Exploit and Prepare: review methods to exploit} environmental uncertainties as well as strategic uncertainties and their long-term operational impact

This theme considered the exploitation of uncertainties in application areas, such as: acoustics and tomography, decision support and decision making, operational effectiveness, adaptive sampling, data fusion, and human factors. It also considered future uncertainties, such as: the impact of 
climate change, multiple futures, environmental challenges in the High North, extreme climates, and events.

Grasso et al. (2012) present a decision support system (DSS) for planning maritime operations. Multiple environmental parameters and their uncertainties are input into the DSS. These uncertainties are propagated through the DSS such that the output not only includes a geographical representation of the recommended course of action (e.g., trafficlight map indicating whether to run an operation) but also includes the confidence levels for the recommended actions. After explaining the theoretical underpinnings of their generic DSS, the authors demonstrate its capabilities to plan underwater glider missions (surfacing and transmitting data) using a field study in which the weather conditions worsen considerably during a 72-h period.

Strode et al. (2012) investigate how the uncertainty in sound speed profiles manifests itself and impacts the performance of a multi-static active sonar sensor network. This is evaluated using the Multistatic Tactical Planning Aid (MSTPA) which has the ability to include three-dimensional temperature and salinity fields, from which sound speed is calculated. Using 100 realizations of the environment perturbed according to the uncertainty estimated from the 3DSE referred to previously, MSTPA was used to estimate signal excess and probability of detection for an active sonar scenario.

Munafò et al. (2011) explore a data-driven adaptive sampling approach for AUVs based on emergent agent behavior techniques and artificial potential functions to achieve a desired goal. The method does not require the explicit minimization of a cost function and easily accommodates constraints such as underwater communication ranges between vehicles.

Incze (2011) explores various Concept of Operations of lightweight AUVs through integration of Commercial OffThe-Shelf hardware components and software development for enhanced on-board Command and Control functions. Shore- and ship-launched scenarios with launch/recovery by a single operator in a one-to-many coordinated survey, on-scene data product generation and visualization, data push to Reach Back Cells for product integration and enhancement, and survey optimization to streamline survey effort and timelines were included in this review.

\footnotetext{
Acknowledgments The conference co-chairs would like to express their gratitude to the organizers and sponsors: the NATO Undersea Research Center (NURC), United States Office of Naval Research Global (ONRG), Springer (publisher of Ocean Dynamics), and Centro Congressi Carispezia (owners of the beautiful Villa Marigola conference venue). Michel Rixen is very grateful to his NURC colleagues for their dedication to the uncertainty challenge. Pierre Lermusiaux is very grateful to the Office of Naval Research for partial support under grants N00014-08-1-0586 (QPE), N00014-08-1-1097 (ONR6.1), and N00014-08-1-0680 (PLUS-SEAS).
}

Open Access This article is distributed under the terms of the Creative Commons Attribution License which permits any use, distribution, and reproduction in any medium, provided the original author(s) and the source are credited.

\section{References}

Bell PS, Osler JC (2011) Mapping bathymetry using X-band marine radar data recorded from a moving vessel. Ocean Dyn 61 (12):2141-2156

Bertotti L, Cavaleri L (2011) The predictability of meteooceanographic events. Ocean Dyn 61(9):1391-1402

Blain CA, Cambazoglu MK, Linzell RS, Dresback KM, Kolar RL (2011) The predictability of near-coastal currents using a baroclinic unstructured grid model. Ocean Dyn 62(3). doi:10.1007/s10236-0110501-9 (this issue)

Breivik Ø, Bekkvik TC, Wettre C, Ommundsen A (2011) BAKTRAK: backtracking drifting objects using an iterative algorithm with a forward trajectory model. Ocean Dyn 62(2):239-252

Brizzolara S, Curtin T, Bovio M, Vernengo G (2011) Concept design and hydrodynamic optimization of an innovative SWATH USV by CFD methods. Ocean Dyn 62(2):227-237

Callies U, Plüß A, Kappenberg J, Kapitza H (2011) Particle tracking in the vicinity of Helgoland, North Sea: a model comparison. Ocean Dyn 61(12):2121-2139

Chiggiato J, Jarosz E, Book JW, Dykes J, Torrisi L et al (2012) Dynamics of the circulation in the Sea of Marmara: numerical modeling experiments and observations from the Turkish straits system experiment. Ocean Dyn 62(1):139-159

Cococcioni M, Corucci L, Masini A, Nardelli F (2012) SVME: an ensemble of support vector machines for detecting oil spills from full resolution MODIS images. Ocean Dyn 62(3). doi:10.1007/ s10236-011-0510-8 (this issue)

Grasso R, Cococcioni M, Chiggiato J, Rixen M (2012) A maritime decision support system to assess risk in the presence of environmental uncertainties: the REP10 experiment. Ocean Dyn 62(3). doi:10.1007/s10236-011-0512-6 (this issue)

Hjelmervik KT, Jensen JK, Østenstad P, Ommundsen A (2011) Classification of acoustically stable areas using empirical orthogonal functions. Ocean Dyn 62(2):253-264

Holman RA, Holland KT, Lalejini DM, Spansel SD (2011) Surf zone characterization from Unmanned Aerial Vehicle imagery. Ocean Dyn 61(11):1927-1935

Holt TR, Cummings JA, Bishop CH, Doyle JD, Hong X et al (2011) Development and testing of a coupled ocean-atmosphere mesoscale ensemble prediction system. Ocean Dyn 61(11):1937-1954

Incze ML (2011) Lightweight Autonomous Underwater Vehicles (AUVs) performing coastal survey operations in REP 10A. Ocean Dyn 61(11):1955-1965

Marmain J, Forget P, Molcard A (2011) Characterization of ocean surface current properties from single site HF/VHF radar. Ocean Dyn 61(11):1967-1979

Mourre B, Chiggiato J, Lenartz F, Rixen M (2011) Uncertainty forecast from 3-D super-ensemble multi-model combination: validation and calibration. Ocean Dyn 62(2):283-294

Munafò A, Simetti E, Turetta A, Caiti A, Casalino G (2011) Autonomous underwater vehicle teams for adaptive ocean sampling: a data-driven approach. Ocean Dyn 61(11):1981-1994

Pecknold S, Osler JC (2011) Sensitivity of acoustic propagation to uncertainties in the marine environment as characterized by various rapid environmental assessment methods. Ocean Dyn 62(2):265-281

Pleskachevsky A, Lehner S, Heege T, Mott C (2011) Synergy and fusion of optical and synthetic aperture radar satellite data for 
underwater topography estimation in coastal areas. Ocean Dyn 61 (12):2099-2120

Shen W, Gurgel K-W, Voulgaris G, Schlick T, Stammer D (2012) Wind-speed inversion from HF radar first-order backscatter signal. Ocean Dyn 62(1):105-121

Strode C, Mourre B, Rixen M (2012) Decision support using the Multistatic Tactical Planning Aid (MSTPA). Ocean Dyn 62(1):161-175
Vousdoukas MI, Wziatek D, Almeida LP (2012) Coastal vulnerability assessment based on video wave run-up observations at a mesotidal, steepsloped beach. Ocean Dyn 62(1):123137

Wadhams P (2012) The use of autonomous underwater vehicles to map the variability of under-ice topography. Ocean Dyn 62(3). doi:10.1007/s10236-011-0509-1 (this issue) 